in vivo $35: 3633-3639(2021)$

doi:10.21873/invivo.12670

\title{
A Rare Cause of Massive Hematuria: Placenta Percreta With Bladder Invasion
}

\author{
RALUCA BALAN ${ }^{1,2}$, VIOREL DRAGOȘ RADU ${ }^{3,4}$, SIMONA-ELIZA GIUȘCA $^{1,5}$, CRISTIAN COSTACHE ${ }^{3,4}$, \\ CONSTANTIN RISTESCU ${ }^{3,4}$, DRAGOȘ PUIA ${ }^{3,4}$, PAVEL ONOFREI ${ }^{6}$, MIRCEA ONOFRIESCU ${ }^{7,8}$, \\ ADINA TĂNASE ${ }^{7,8}$, RĂZVAN POPOVICI $^{7,8}$, DEMETRA SOCOLOV $^{7,8}$ and IRINA-DRAGA CĂRUNTU ${ }^{1,5}$ \\ ${ }^{1}$ Department of Morpho-Functional Sciences I, \\ University of Medicine and Pharmacy "Grigore T. Popa”, Iași, Romania; \\ ${ }^{2}$ Department of Pathology, Clinical Hospital of Obstetrics and Gynecology "Elena Doamna”, Iaşi, Romania; \\ ${ }^{3}$ Department of Surgery, University of Medicine and Pharmacy "Grigore T. Popa”, Iași, Romania; \\ ${ }^{4}$ Department of Urology, Clinical Hospital “Dr. C. I. Parhon”, Iași, Romania; \\ ${ }^{5}$ Department of Pathology, Clinical Hospital "Dr. C. I. Parhon”, Iași, Romania; \\ ${ }^{6}$ Department of Morpho-Functional Sciences II, University of Medicine and Pharmacy “Grigore T. Popa”, Iași, Romania; \\ ${ }^{7}$ Department of Mother and Child Medicine, University of Medicine and Pharmacy "Grigore T. Popa”, Iași, Romania; \\ ${ }^{8}$ Department of Obstetrics and Gynecology, Clinical Hospital of Obstetrics and Gynecology “Cuza-Vodă”, Iaşi, Romania
}

\begin{abstract}
Background/Aim: Placenta percreta is a rare event, but it poses serious problems due to potential hemorrhagic events. We report a particular case of placenta percreta with massive hematuria due to maternal bladder invasion, and describe the surgical protocol performed that resulted in an excellent outcome. Case Report: A 33-yearold patient, at $27^{\text {th }}$ weeks gestational age, presented in the emergency room of the Urology Department with urinary blood clot acute retention, because of massive hematuria from a placenta percreta with bladder invasion. After extracting the clots from the bladder, and coagulation of an area of venous ectasies of the posterior wall, hematuria ceased, but appeared after two days, necessitating again the bladder clots removal and coagulation. A surgical team with gynecologists, urologists, anesthesiologists and a neonatologist was composed, and after bilateral ureteral double J insertion, cesarean section was performed followed by hemostatic hysterectomy and partial cystectomy, bilateral internal iliac artery ligature and repair of the bladder wall. The postoperative evolution was without incidents; the Foley catheter was removed in the $14^{\text {th }}$ postoperative day. Conclusion: In the context of a massive hematuria of a
\end{abstract}

This article is freely accessible online.

Correspondence to: Viorel Dragoș Radu, Str. Universităţii 16, Iași, 700115, Romania. Tel: +40740060160, e-mail: vioreldradu@ yahoo.com and Pavel Onofrei, Str. Universității 16, Iași, 700115, Romania. Tel: +40 742264048, e-mail: onofrei.pavel@gmail.com

Key Words: Placenta percreta, bladder invasion, massive hematuria. pregnant woman, the urologist must always consider a diagnosis of complicated placenta percreta.

Placenta percreta represents the most severe form of abnormal trophoblastic adherence beyond the decidua basalis, among the three representatives of the placenta accreta spectrum (PAS), a rare condition with reported incidence of $1 / 500$ to $1 / 2 / 500$ pregnancies $(1,2)$. PAS includes $75-80 \%$ cases of placenta accreta vera (less than $50 \%$ myometrial invasion by the trophoblast), $17 \%$ cases of placenta increta (more than $50 \%$ myometrial invasion by the trophoblast), and $5 \%$ cases of percreta (invasion of uterine serosa and neighboring pelvic organs) $(2,3)$. Moreover, the abnormal adherence can be complete (throughout the entire placenta), partial (limited to only one or more cotyledons), or focal (in isolated areas) (4).

More than 2 caesarean sections (CS), including shorter intervals between previous CS and current pregnancy (less than 2 years), and concurrent placenta praevia (in $75 \%$ of cases) are the most common known risk factors, followed by advanced maternal age, multiparity, endometritis, hypertension, assisted reproductive technology, submucosal leiomyomas, other uterine surgeries and anomalies (poor quality of scarring and CS performed on long time ruptured membranes leading to chorioamniotitis), smoking $(3,5-9)$. Because of the rise in the numbers of CS performed in recent years, the risk of PAS disorders is increasing up to 10 times in the last 50 years $(10,11)$.

The information regardless of treatment of the placenta percreta with bladder invasion are limited, because of the exceptional rarity of this event. Approximatively 70 cases are reported as case presentation, the largest series comprising 54 patients (12). 


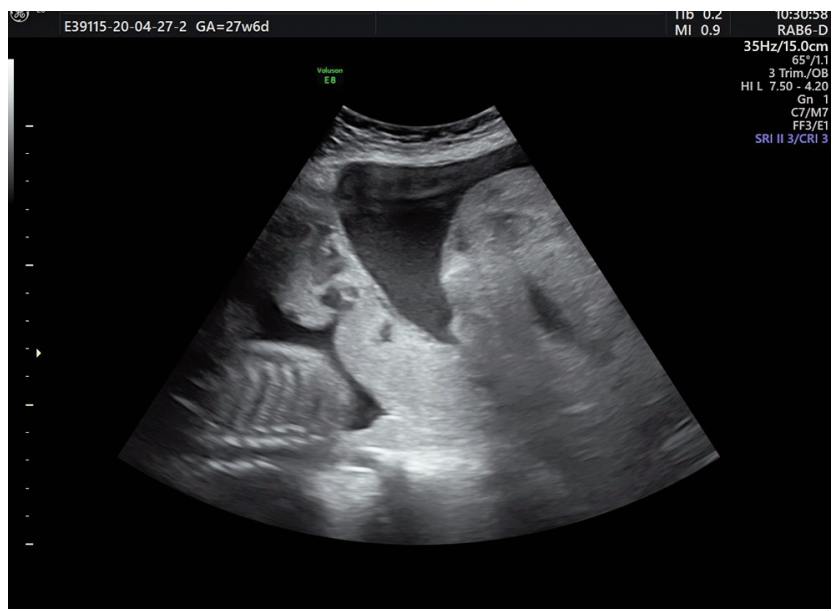

Figure 1. Ultrasound examination of placenta praevia percreta: on the right side, a blood clot; on the left side, fetus with visible costal grid; on the center, placenta.

The suspicion or the early diagnosis of PAS disorders is based on the theoretical knowledge of the risk factors in relation with the patient's obstetrical and general medical history. Supplementary, the ultrasonography or magnetic resonance imaging (MRI), when ultrasound cannot provide specific data, contribute to the clinical diagnosis.

Most placenta accreta are asymptomatic to term and if diagnosed by ultrasound during pregnancy, can be scheduled in special centers and resolved in multidisciplinary teams (2, $3,13)$. The review of the literature shows that it is mandatory for the placenta percreta to be diagnosed early during pregnancy, because of the serious fetal and maternal implications of this condition. These pregnancies can be complicated at birth, during the detachment of the placenta from the uterus, producing massive hemorrhage and trauma to neighboring organs in which the invasion occurred (bladder and rarely the ureters, rectum, iliac vessels). After birth, postpartum hemorrhage manifested by vaginal bleeding $(10,14)$ and more rarely hematuria $(8,11,12,15$, 16) can appear. Therefore, an optimal therapeutic strategy is mandatory $(3,13)$.

Life-threatening hematuria, a fatal complication, manifests in the $25 \%$ of the cases of placenta percreta, in the second trimester, when the placenta invades the maternal bladder wall, which requires premature evacuation, leading to challenges in surgical management $(2,3,13,16)$. Maternal and fetal mortality in cases of percreta with hematuria is high, especially because of intraoperative and postoperative complications (up to $5 \%$ maternal deaths) (12), but our case was successfully resolved by cesarean section - hysterectomy with bilateral ligation of hypogastric arteries.

Although the evidence of the urinary bladder involvement is mostly intraoperatively confirmed, during the delivery (2),

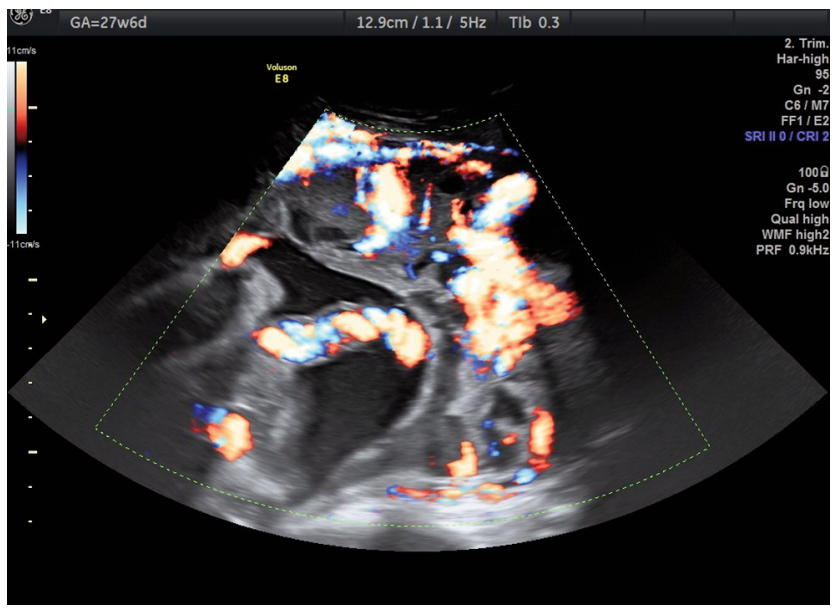

Figure 2. Ultrasound Doppler examination: absence of vascularization in the intravesical solid mass.

the gold-standard certification comes only after the histopathological examination (3). We present a particular case of placenta percreta with massive hematuria due to maternal bladder invasion and the surgical protocol performed, with excellent outcome.

\section{Case Report}

A 33-year-old woman, at 27 weeks gestational age, secundiparous, presented into the emergency room of the Urology Department with intermittent macroscopic hematuria and acute urinary retention. The COVID-19 test was negative. The case history revealed no specific follow-up of the pregnancy, due to a low socio-economical status of the gravida.

The ultrasound obstetrical examination confirmed a good evolution of the fetus but described a solid mass in the bladder of 13.68/9.74 cm, without Doppler vascularization (Figure 1 and Figure 2). The problem of differential diagnosis between a solid bladder tumor and a clot has been raised, but the localization of the placenta praevia, with anterior extension on the uterine scar and the absence of Doppler vascularization at the level of the intravesical solid mass, supported the second hypothesis.

Hemoglobin $(\mathrm{Hb})$ at admission was $11.4 \mathrm{~g} / 100 \mathrm{ml}$. In the first hours after admission, she developed massive hematuria, with urinary acute retention due to intravesical clots, with a drop of $\mathrm{Hb}$ till $5 \mathrm{~g} / 100 \mathrm{ml}$. Emergency cystoscopy and evacuation of the clots were performed. On the posterior wall of the bladder, venous ecstasies were seen with active bleeding, which were coagulated. Urine became clear on urethral Foley catheter, with no need of bladder irrigation. Four units of isogroup, iso-Rh were administrated. After $48 \mathrm{~h}$, the Foley catheter was removed due to clear aspect of the urine. The 


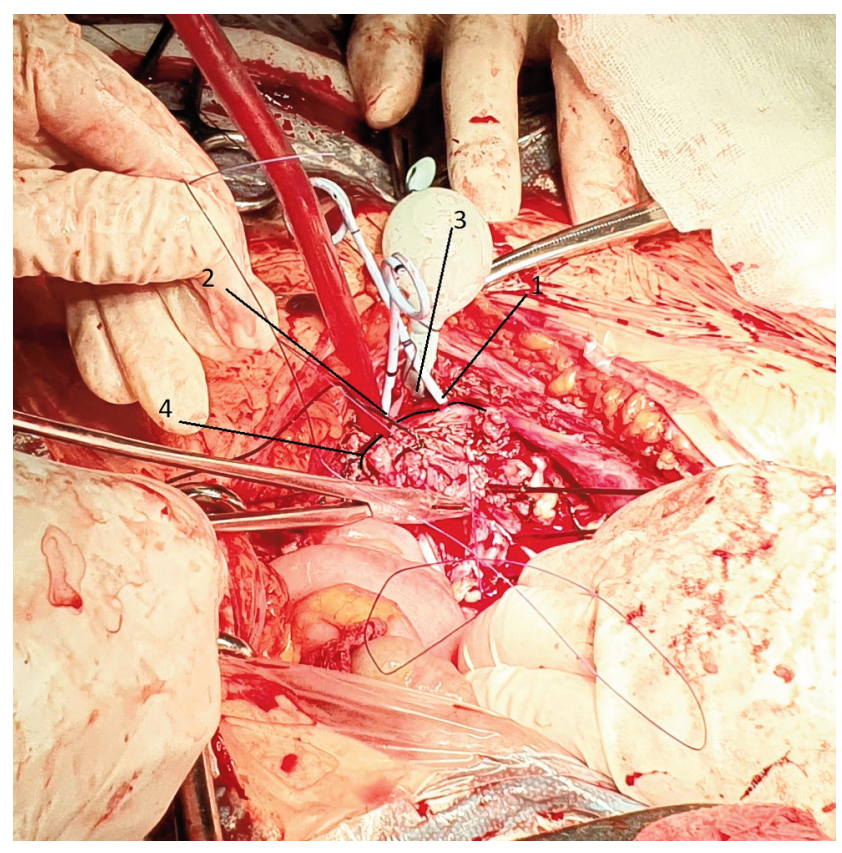

Figure 3. Intraoperative aspects illustrating the surgical procedure: 1) right ureteral double J stent; 2) left ureteral double J stent; 3) bladder neck; 4) ruptured bladder wall.

patient developed once again, with spontaneous urination, gross hematuria, with a drop of $\mathrm{Hb}$ from 7 to $5.3 \mathrm{~g} / 100 \mathrm{ml}$. A new cystoscopy was performed, with clots extraction, coagulation of the same venous ecstasies of the posterior wall and administration of three units of blood. The urine cleared and a Foley catheter was reinserted. The team composed of gynecologists, urologists, anesthesiologists and a neonatologist decided to perform the cesarean section after $72 \mathrm{~h}$ from the last intervention. First, bilateral $7 \mathrm{CH}$ ureteral double $\mathrm{J}$ stents were inserted, after which cesarean section was performed. The child was born with 980 grams and Apgar 7, with a good postoperative evolution in our tertiary neonatal department. Intraoperatively, placenta was attached to the posterior wall of the bladder, and hemorrhage started during decollation of the uterus, vagina and bladder, necessitating hemostatic hysterectomy and bilateral ligature of the internal iliac arteries. During hysterectomy, the bladder wall was opened, to permit the extraction of the placenta with a portion of invaded bladder wall (Figure 3 and Figure 4). After thorough hemostasis at the level of vaginal stump, broad ligament and the bladder, cystotomy was sutured with running uninterrupted 2-0 Vicryl, in one plane. At the end, the tightness of the suture was verified with methylene blue. Intraoperatively 8 units of blood were necessary. Forty-eight hours after the operation, $\mathrm{Hb}$ was 7.6 $\mathrm{g} / 100 \mathrm{ml}$. Postoperative evolution was uneventful, the patient was discharged in the $8^{\text {th }}$ postoperative day, the Foley catheter was removed ambulatory in the $14^{\text {th }}$ postoperative day.

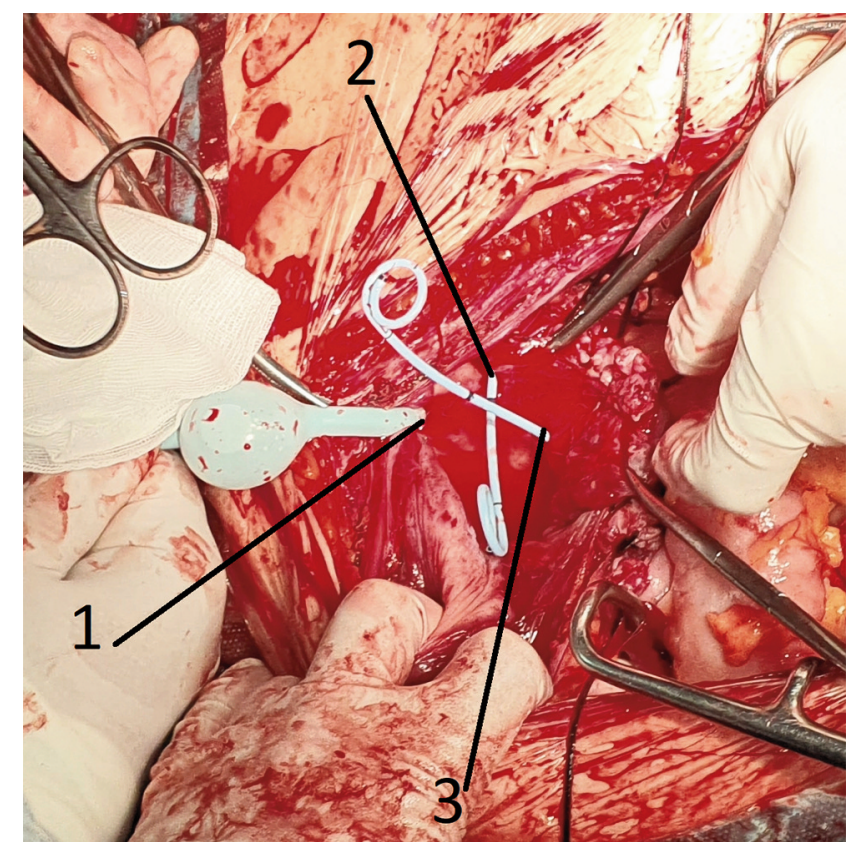

Figure 4. Detailed image of the bilateral 7CH ureteral double J stents: 1) bladder neck; 2) right ureteral double J stent; 3) left ureteral double J stent.

The placenta percreta was documented on the hysterectomy specimen, which was grossly and microscopically examined, revealing stem and terminal placental villi completely invading the whole thickness of gestational myometrium, which was globally thinned, as well as the uterine serosa, penetrating focally the external surface. The placental villi were attached to myometrial layer, and decidua basalis was reduced or absent (Figure 5). Intermediate trophoblast dissected the myometrium, involving also the arterial wall and contributing to their remodeling. Two of the examined fragments presented villi and intermediate trophoblast lining the serosa of the urinary bladder, reaching the external half of the bladder muscularis propria, with focally involvement of the vascular walls (Figure 6). The placenta praevia was pathologically diagnosed by the trophoblast invasion in the lamina propria and fibromuscular layer of the isthmus and endocervical internal os (Figure 7).

Ethics approval and consent to participate. The study has been approved by the Ethics Committee of the Clinical Hospital "Dr. C. I. Parhon" Iaşi, based on the patients' informed consent.

Consent for publication. Written informed consent was obtained from the patient for publication of this case report and the accompanying images. 


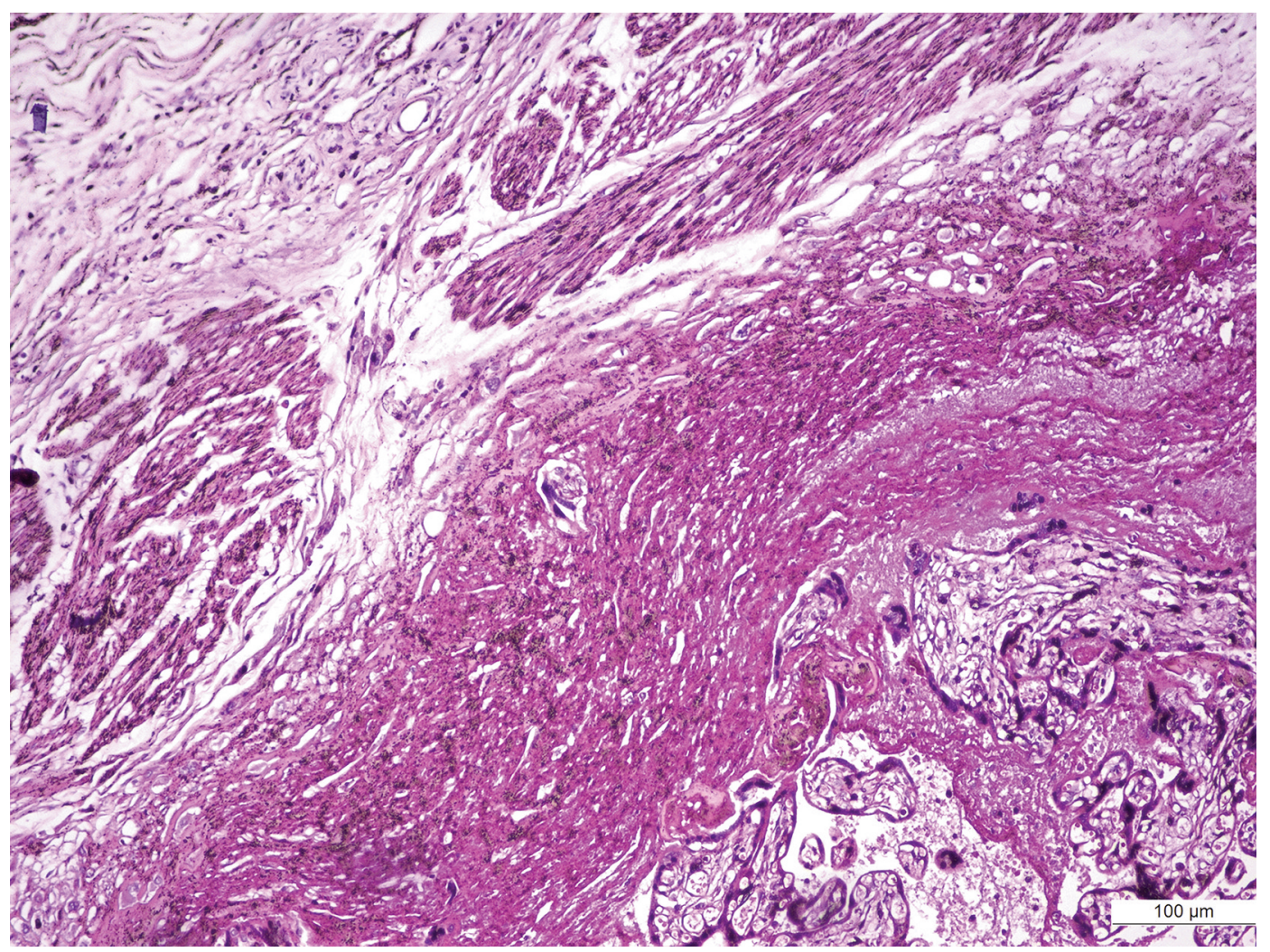

Figure 5. Myometrial involvement by the placental villi (haematoxylin and eosin staining, magnification $\times 10$ ).

\section{Discussion and Conclusion}

Unlike normal pregnancies, in which trophoblastic invasion ceases when the cells reach the spongiosum layer of the decidua (Nitabuch's layer) (17), in PAS the abnormal adherence of the trophoblastic cells produce damage of the decidua basalis, with thoroughly gradual invasion of the uterine wall (3).

Although placenta percreta is the least frequent form of PAS and can sustain a full-term pregnancy with normal fetal development, it is considered the most severe of the three conditions, sometimes causing premature labor or heavy peripartum bleeding, and moreover, greater complications if diagnosed intraoperatively or if trophoblastic invasion involves neighboring organs or structures $(4,5)$. Within this context, the invasion of the urinary bladder with subsequent life-threatening hematuria represents a rare complication of placenta percreta.

To the best of our knowledge, only Washecka et al. (12), in 2002, reported a series of 54 cases after a review of the literature, in which 17 (31\%) patients presented hematuria, reporting 3 maternal and 17 fetal deaths. There are also few case reports that present peculiar aspects of the surgical management of placenta percreta with bladder wall involvement $(8,10,11,15,16)$. Thus, we presented a successful treatment of a placenta percreta associated with placenta praevia and complicated with massive hematuria, with a very good outcome.

The best therapeutic option is still controversial $(9,13)$. In case of placenta percreta with bladder wall involvement, the elective approach is cesarean hysterectomy, followed by cystotomy or partial cystectomy, with the risk of urinary fistula and prolonged catheterization $(3,11,13)$.

In our case, primary hysterectomy was mandatory, as reported in other studies $(2,3,10,14,15)$, because uterus preservation is associated with the hemorrhagic risk, even months after CS. We also proposed, for a good hemostasis, bilateral internal iliac artery ligature, strategy already reported in the literature (5). To avoid intraoperative ureteral injuries, we inserted bilateral, ureteral double J stents (10, 13). Our multidisciplinary team composed of gynecologists, urologists and neonatologists achieved a good postoperative outcome, with a progressive recovery of mother and the improvement of the newborn health.

Because of the increasing frequency of short-term and long-term consequences due to radical surgical techniques 


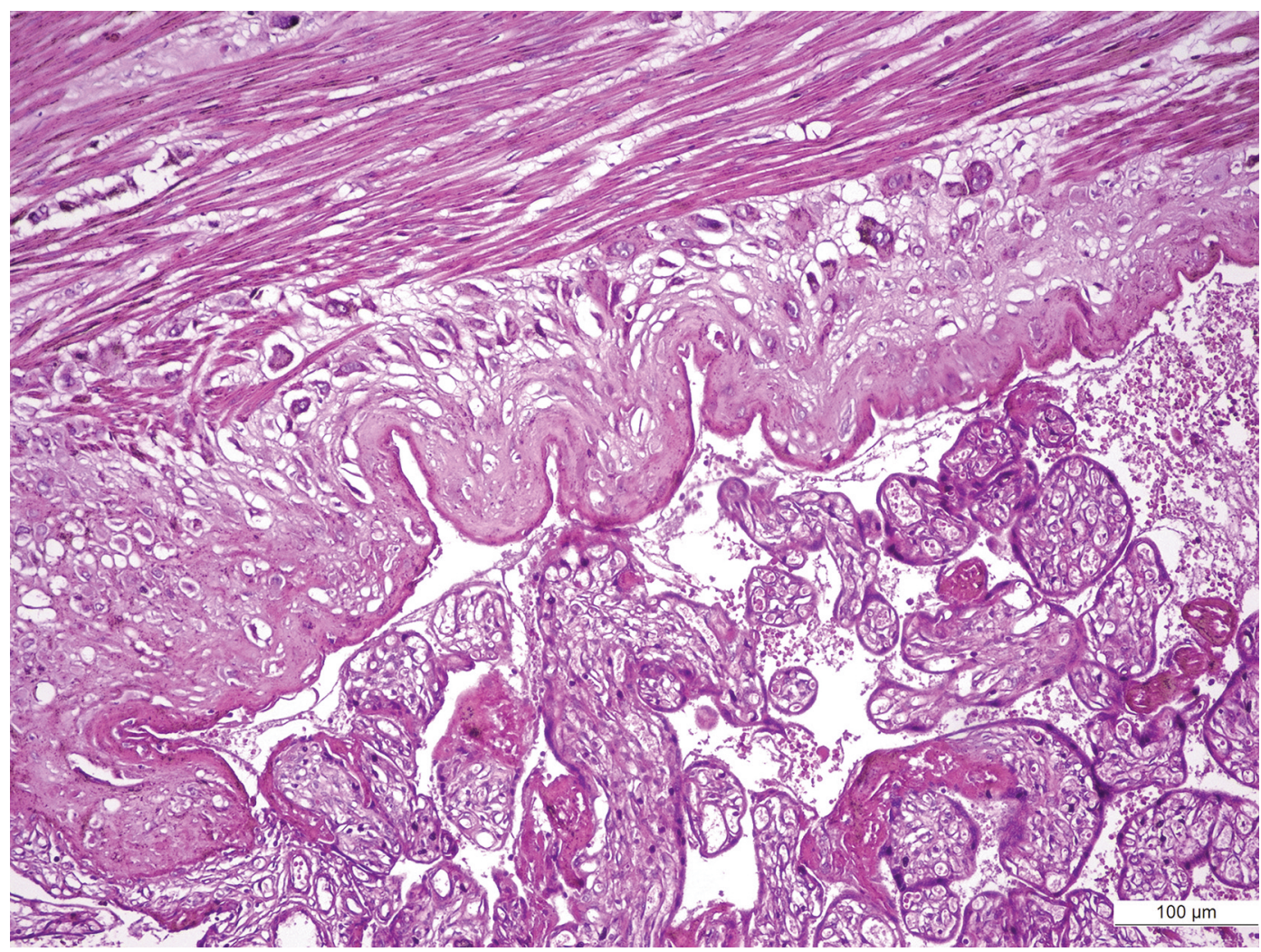

Figure 6. Bladder wall involvement by villi and intermediate trophoblast (haematoxylin and eosin staining, magnification $\times 10$ ).

used in the management of maternal complications of the PAS, the surgical strategy has progressively evolved towards more conservative techniques, such as: the "triple $\mathrm{P}$ procedure" (ultrasound perioperative placental localization, pelvic devascularization, and placental non-separation), intentional placental retention or partial myometrial excision $(3,13)$. The in situ maintenance of the placenta adherent to the uterine and urinary bladder wall, respectively, implies the use of methotrexate for placental involution (spontaneously resorbed in 9-12 months), as well as a careful follow-up at least 20 weeks because of the high risk of sepsis, delayed hematuria and massive hemorrhage, with subsequent peripartum hysterectomy $(3,12,13)$. If the abnormal placental penetration is partial or focal, a possible surgical option is one-step conservative surgery, that consists in the resection of myometrium and bladder tissues invaded by placenta, concomitantly with the reconstruction of the affected areas $(3,13)$. However, this procedure presents several disadvantages, namely: sepsis, multiple organ failure, intravascular disseminated coagulopathy, and vesicovaginal fistula $(3,13)$.

The key particularity of our case is the existence of only one previous CS, these varieties of placenta percreta appearing on multiscar uteri, the risk increasing geometrically with the number of scars. The presence of the placenta praevia, as an important risk factor of placental accretion, associated in our case with the placenta percreta is also remarkable, considering that previous CS was more than two years apart (5 years ago) and without scarring defects. Moreover, no data of pregnancy evolution were documented, as patient addressed directly to the Urology service for intermittent hematuria and acute urinary retention. Another challenge of the case was featured by the fact that abdominal ultrasound, which revealed a solid intravesical mass of $13.68 / 9.74 \mathrm{~cm}$, had to perform the differential diagnosis between a solid bladder tumor and a giant blood clot. The conservative therapeutic option could not be applied in our case, because it would not have stopped the hematuria. It is worth mentioning that the attempt to coagulate by cystoscopy the vessels of the bladder mucosa developed by the abnormal placental invasion failed, by the hematuria recurrence, after the first suppression of the Foley catheter. A distinct feature of our report consists in the histological documentation of the abnormal placentation into the muscular layer of the urinary bladder, as the literature provides few typical microscopic images for this condition. 


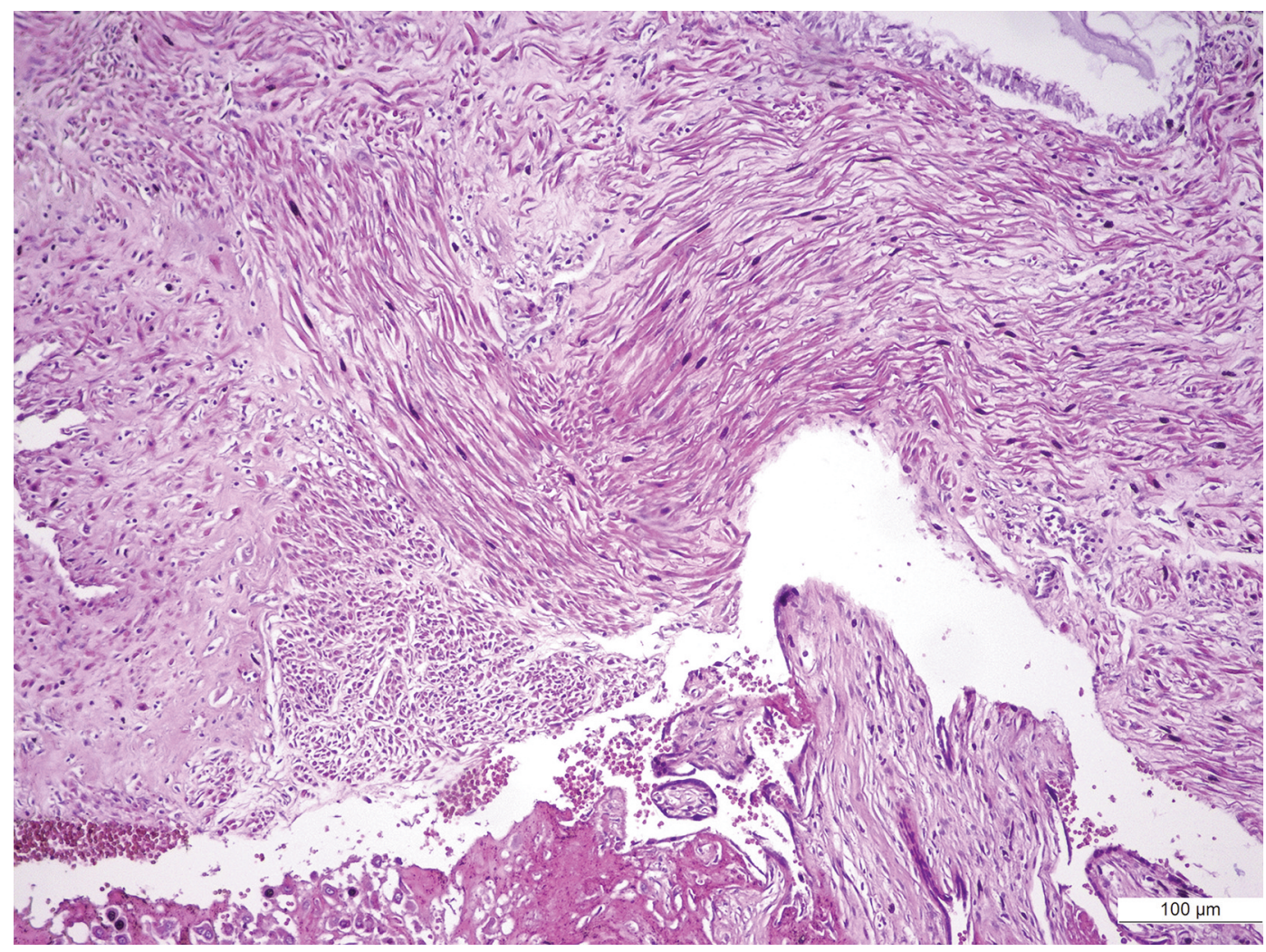

Figure 7. Endocervical involvement by the trophoblast (haematoxylin and eosin staining, magnification $\times 10$ ).

To conclude, placenta percreta associated with bladder invasion is a rare but serious, life-threatening problem. The urologist should consider a massive hematuria of a pregnant woman as key diagnostic feature for placenta percreta.

\section{Conflicts of Interest}

The Authors declare no conflicts of interest.

\section{Authors' Contributions}

VDR, RA, SEG and PO have designed and have written the report, including the literature search; VDR, CC, CR, DP, MO and AT have performed the surgery; DS and RP have performed the ultrasound obstetrical examination; RA, SEG and IDC have performed the histopathological examination; DS and IDC have revised critically the manuscript for important intellectual content. All authors have read and approved the final manuscript.

\section{References}

1 Wu S, Kocherginsky M and Hibbard JU: Abnormal placentation: twenty-year analysis. Am J Obstet Gynecol 192(5): 1458-1461, 2005. PMID: 15902137. DOI: 10.1016/j.ajog.2004.12.074

2 Konijeti R, Rajfer J and Askari A: Placenta percreta and the urologist. Rev Urol 11(3): 173-176, 2009. PMID: 19918343.
3 Piñas Carrillo A and Chandraharan E: Placenta accreta spectrum: Risk factors, diagnosis and management with special reference to the Triple $\mathrm{P}$ procedure. Womens Health (Lond) 15: 1745506519878081, 2019. PMID: 31578123. DOI: 10.1177/ 1745506519878081

4 Gersell D and Kraus F: Diseases of the placenta. Blaustein's Pathology of the Female Genital Tract: 999-1073, 2020. DOI: 10.1007/978-1-4419-0489-8_19

5 Okunowo AA, Ohazurike EO and Habeebu-Adeyemi FM: Undiagnosed placenta praevia percreta: A rare case report and review of management. Niger Postgrad Med J 26(1): 61-64, 2019. PMID: 30860201. DOI: 10.4103/npmj.npmj_191_18

6 Bartels HC, Postle JD, Downey P and Brennan DJ: Placenta accreta spectrum: A review of pathology, molecular biology, and biomarkers. Dis Markers 2018: 1507674, 2018. PMID: 30057649. DOI: 10.1155/2018/1507674

7 Kyozuka H, Yamaguchi A, Suzuki D, Fujimori K, Hosoya M, Yasumura S, Yokoyama T, Sato A, Hashimoto K and Japan Environment and Children's Study (JECS) Group: Risk factors for placenta accreta spectrum: findings from the Japan environment and Children's study. BMC Pregnancy Childbirth 19(1): 447, 2019. PMID: 31775687. DOI: 10.1186/s12884-019-2608-9

8 Abbas F, Talati J, Wasti S, Akram S, Ghaffar S and Qureshi R: Placenta percreta with bladder invasion as a cause of life threatening hemorrhage. J Urol 164(4): 1270-1274, 2000. PMID: 10992378.

9 Paniza LR, Durán SR, Hita M, Paniza MR, Villaverde GR, Armenteros MB and Prieto AP: Placenta percreta a case study 
and literature review. Int J Pregnancy Child Birth 4(5): 232-235, 2018. DOI: 10.15406/ipcb.2018.04.00114

10 Smith ZL, Sehgal SS, Van Arsdalen KN and Goldstein IS: Placenta percreta with invasion into the urinary bladder. Urol Case Rep 2(1): 31-32, 2014. PMID: 26955539. DOI: 10.1016/ j.eucr.2013.11.010

11 Koukoura O, Lialios G, Garas A, Sveronis G, Nidimos A, Gkorezi I, Alevra Z, Tzortzis V, Oeconomou A, Zachos I and Daponte A: Macroscopic hematuria due to placenta percreta: Report of two cases and short review. Case Rep Obstet Gynecol 2017: 9863792, 2017. PMID: 28698813. DOI: 10.1155/ 2017/9863792

12 Washecka R and Behling A: Urologic complications of placenta percreta invading the urinary bladder: a case report and review of the literature. Hawaii Med J 61(4): 66-69, 2002. PMID: 12050959.

13 Tillu N, Savalia A, Patwardhan S and Patil B: Placenta percreta with bladder invasion: The armamentarium available in its management. Urol Ann 11(3): 324-327, 2019. PMID: 31413516. DOI: 10.4103/UA.UA_84_18

14 Sijanović S, Rubin M, Topolovec Z, Vidosavljević D and Sijanović I: Placenta previa percreta with bladder invasion. Med Glas (Zenica) 8(1): 66-68, 2011. PMID: 21263399.
15 Aho AJ, Pulkkinen MO and Vähä-Eskeli K: Acute urinary bladder tamponade with hypovolemic shock due to placenta percreta with bladder invasion. Case report. Scand J Urol Nephrol 19(2): 157-159, 1985. PMID: 4059882. DOI: 10.3109/ 00365598509180248

16 Takai N, Eto M, Sato F, Mimata H and Miyakawa I: Placenta percreta invading the urinary bladder. Arch Gynecol Obstet 271(3): 274-275, 2005. PMID: 15791478. DOI: 10.1007/s00404004-0651-9

17 Silver RM and Barbour KD: Placenta accreta spectrum: accreta, increta, and percreta. Obstet Gynecol Clin North Am 42(2): 381402, 2015. PMID: 26002174. DOI: 10.1016/j.ogc.2015.01. 014References

Received July 21, 2021

Revised August 27, 2021

Accepted September 7, 2021 